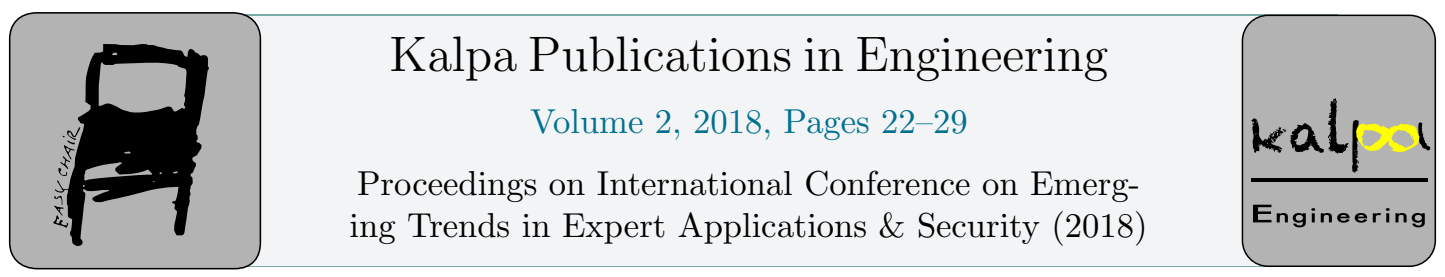

\title{
Comparative Analysis of TCP, SCTP and MPTCP in Transport Layer of Wireless Sensor Networks
}

\author{
Geerija Lavania $^{1,2 *}$ and Preeti Sharma ${ }^{1,2}$ Richa Upadhyay ${ }^{1,2}$ \\ ${ }^{1}$ EasyChair \\ ${ }^{2}$ Department of Computer Science,JECRC. \\ girija47lavania@gmail.com, sharma.preeti251.ps@gmail.com, richaupad \\ hyay.csel jecrc.ac.in
}

\begin{abstract}
The wireless sensor network is the network that has large number of sensor nodes that are connected to each other. The wireless nodes sense the event and forward packets to the destination node. A transport layer handles congestion and packet loss recovery for reliable data transfer in WSN. There exist several protocols at the transport layer in WSN for reliable data transfer like ESRT, ATP, Tiny TCP/IP, PORT, CTCP, RTMC, DCDD, RETP etc. Each protocol has its merits and demerits. Traditional network uses TCP and UDP protocol at the transport layer. In WSN, these are not suitable. In this work, the TCP, SCTP and MPTCP are compared in the wireless sensor network environment. The wireless network with packet loss is considered. From the comparative analysis, we get the result that MPTCP gives the better performance than TCP and SCTP in the wireless sensor network.
\end{abstract}

Keywords: TCP, SCTP, MPTCP, Reliability WSN

\section{Introduction}

\subsection{Wireless Sensor Network}

Large number of sensors is connected with each other to perform certain task. A Wireless Sensor Network (WSN) is the network of hundreds of nodes that has sensors and used for monitoring the events in a certain geographic area [1]. The sensors are used to monitor different conditions for example sound, pressure, temperature etc. in the environment [2]. It consists of Data Acquisition

\footnotetext{
${ }^{*}$ Masterminded EasyChair and created the first stable version of this document
} 
Network (DAN) and a Data Distribution Network (DDN). Both are managed by a Management Centre. A wireless SN is low power device that has the following components:
a) Processor
b) Memory
c) Transceiver
d) Sensors
e) Battery for power supply

\subsection{Layer}

1.2.1. Physical Layer:It deal with the physical connectivity. It is responsible for transmission, reception and modulation. The possibility of error rate at the physical layer is high and time varying in a wireless environment. The Energy consumed to run the radio circuitry is fixed whereas the energy consumed to transmit the data can vary based on channel loss, interference, and transmission distance.Energy-efficient modulation techniques should minimize both transmission and circuit energy [5].

1.2.2. Data link Layer: It is responsible for data frame, frame control. It concerned with the data transfer between two nodes that share the same link. Medium access and error control. Error detection and correction services are also provided by the data-link layer as well as the transport layer For the recovery in WSN, automatic repeat request (ARQ), forward error correction (FEC), hybrid ARQ (HARQ), simple packet combining (SPaC) etc. techniques used [5].

1.2.3. Network Layer: It is responsible for routing of data across the network from source to destination. IP based protocol is not used in the WSN. Due to these constraints, a sensor network's lifetime is long. The network protocol concern with efficiency, fault tolerance, fairness and security [5].

1.2.4. Transport Layer: It is responsible for the reliability and quality of data at source and sinks and ensures QoS. Due to the packet loss, energy will be wasted and it degrades the quality of service (QoS) [5].

1.2.5.Application Layer: It is responsible for application and user related task. It Provide software for different applications that translate the data in a human readable format [7].

\subsection{Design Issues of Transport Control Protocols}

Following factors are considered for the transport control protocol for WSNs [9]:

It should provide congestion control mechanism and give the guarantee of reliability. The most data streams are flowed from sensor nodes to sink in WSNs, so congestion occurs at the sink. Then the transport protocol for WSNs should have mechanism for packets loss recovery such as ACK and Selective ACK [4] used in TCP protocol so as to guarantee reliability. WSN only need to correctly receive packets from a certain area not every sensor nodes in particular area, or some ratio of successful transmission from a sensor node. 


\section{Literature survey}

The reliability and congestion control in transport layer is an important factor in wireless sensor network. Various techniques are proposed by researchers to improve the reliability and congestion control. Some of them are described below.In 2003, Sankarasubramaniam et al. gave the ESRT. Event-to-Sink Reliable Transport (ESRT) [1] provides the upstream event reliability. It also congestion control when avoiding the dropping of packets. This creates minimum energy consumption. In traditional method of packet reliability, single packet is retransmitted, until the Acknowledgment (ACK) is received from sink node, but the ESRT protocol defines event reliability as the received packet rate associated to the particular sensing process. In 2003, Sundaresan et al proposed ATP [11]. Ad-hoc Transport Protocol (ATP) is designed to improve the performance of TCP in the wireless network. Due to lack of fixed infrastructure, mobility, shared channel resources and limited bandwidth features of Ad hoc network, it is different from the traditional network. In 2004, Dunkels et al., proposed Tiny TCP/IP [12]. Tiny TCP/IP is the modified version of TCP/IP protocol. Tiny TCP/IP provides four methods to use TCP/IP protocol for WSN: spatial IP address assignment, shared context header compression, application overlay routing, and Distributed TCP Caching (DTC). In spatial IP address assignment, every sensor node uses its spatial location to construct the IP address. In 2005, Zhou and Lyu, proposed PORT [13]. Price-Oriented Reliable Transport (PORT) is designed for upstream reliable, congestion control and power efficient transport layer protocol.PORT protocol provides an in-network congestion mechanism to improve traffic dynamically. PORT uses two mechanisms that ensure reliability. In 2005, Iyer et al., proposed STCP [1]. Sensor Transmission Control Protocol (STCP) is an end-to-end upstream transport layer protocol which provides end-to-end reliability as well as congestion control mechanism. In year 2007, Tezcan and Wang, designed ART [14]. Asymmetric and Reliable Transport (ART) is designed to support upstream end to-end event reliability, upstream congestion control and downstream end-to-end query reliabilityin 2007, Kim et al. designed Flush [15] to provide reliable transport protocol for wireless sensor networks. It provides the end-to-end reliability, reduces transfer time, and adapts to timevarying network conditions. It uses sink-initiated control protocol to coordinate transfers, with end-toend selective Negative Acknowledgments (NACK) and retransmissions to provide reliability.In 2007, Braun et al., designed TSS [17].

\section{Proposed work}

A sensor network is defined with vast number of tiny sensor that performs the short distance communication. Because of this multipath communication in smaller network, the congestion over the network increases. The TCP in such network results the packet drop and energy loss. In this present work a parameter-based prioritization approach is defined to perform the reliable communication over the network. The work is about to perform the comparative study in TCP, SCTP and MPTCP.

\subsection{Problem Definition}

A sensor network is the most essential private and public area network used by many applications. The major advantage of this network is its self-motivated topology. This kind of network is defined in different ways for different kind of nodes. These network types actually differ respective to network size, shape, protocol, battery power and the criticality. The analysis of work is done under the packet loss, throughput, bit rate etc. 


\subsection{Significance of Work}

The proposed work will give the following benefits.

a) The scenario defined for nodes localization, the energy specification and consumption parameters for network sensor nodes.

b) The work also includes the comparative analysis of presented approach with traditional protocol.

\subsection{Objectives}

The objectives associated with presented work are given here under:

a) The objective of work is to implement the WSN in NS2 environment.

b) The objective of work is to perform the comparative study of TCP, SCTP andMPTCP under different parameters.

c) The objective of work is to compare the results.

\subsection{Research Design}

The major problems of sensor network are the nodes localization and the communication over the network. The work includes two main phases, one for the neighbor node analysis and prioritization under parametric analysis and other for the reliable and effective data communication mechanism. The parameters considered in this work are energy, distance, communication rate and communication delay.

\subsection{Proposed Work}

In this present work, a comparison to the existing Transport layer is done under the energy parameter specification. In this work, TCP, SCTP and MPTCP are used and analyze the work under different parameter.

\subsection{Network Simulators}

In simulation, a statistical model is built to reproduce the quality of a phenomenon, system or process often using a computer in order to information or solve problems. Nowadays, there are many network simulators that can simulate the WSN.

\section{Result analysis}

\subsection{Parameter Used}

Here the basic parameters of the proposed work are presented respective to the simulation environment. The system is implemented on Fedora Environment with NS2 simulator and XGraph is used as the tool for graph analysis. 


\begin{tabular}{|c|l|c|}
\hline S. No. & \multicolumn{1}{|c|}{ Parameter } & Value \\
\hline 1. & Number of Nodes & 25 \\
\hline 2. & Topography Dimension & $50 \mathrm{~m} \mathrm{x} 50 \mathrm{~m}$ \\
\hline 3. & Traffic Type & CBR \\
\hline 4. & MAC Type & $802.11 . \mathrm{Mac}$ Layer \\
\hline 5. & Packet Size & 512 bytes \\
\hline 6. & Mobility Model & Random Way Point \\
\hline 7. & Antenna Type & Omni directional \\
\hline 8. & Routing Protocol & AODV \\
\hline 9. & Transport Protocol & LL \\
\hline 10. & Link Layer type & 50 \\
\hline 11. & Max packet in ifq & \\
\hline
\end{tabular}

Table 4.1:Parameter used

\subsection{Analysis of Results}

\subsubsection{Wireless Sensor Network Setup}

The wireless sensor network is designed in the NS-2. The model network is shown in figure 4.1. The network comprising of 25 wireless sensor nodes is constructed in the NS-2 simulator with the use ofTCL script in the topological boundary area of $50 \mathrm{~m} \times 50 \mathrm{~m}$. The position of the nodes is defined in terms of $\mathrm{X}$ and $\mathrm{Y}$ coordinates values. A NS2 application will be used to generate sample data.

\subsubsection{WSN using TCP, SCTP and MPTCP with Packet Loss:}

The communication is performed in the wireless sensor network using TCP with packet loss. Some packets don't reach at the destination due to loss. The performance graph for WSN using TCP with packet loss is shown in figure 4.2.

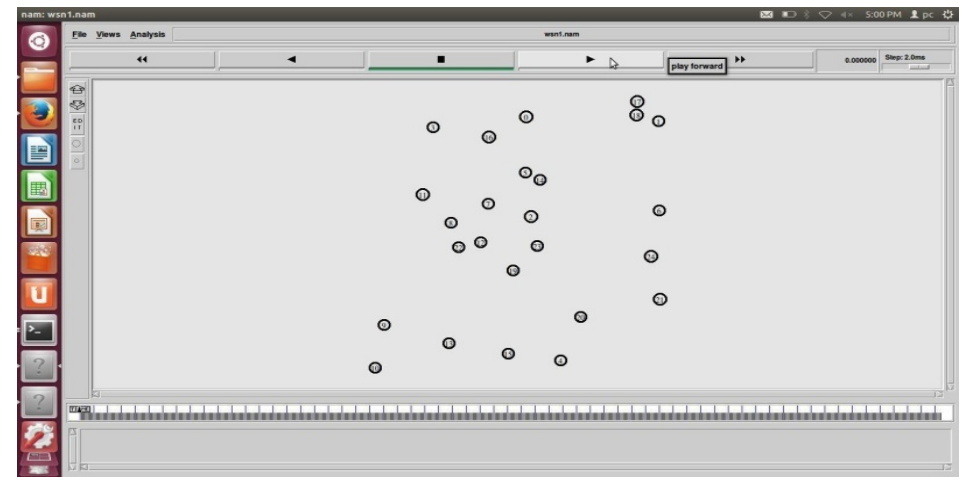

Figure 4.1:Graph for WSN using TCP with packet loss

Some packets don't reach at the destination due to loss. Loss may occur due to any reason. Theperformance graph for WSN using SCTP with packet loss is shown in figure 4.3. 


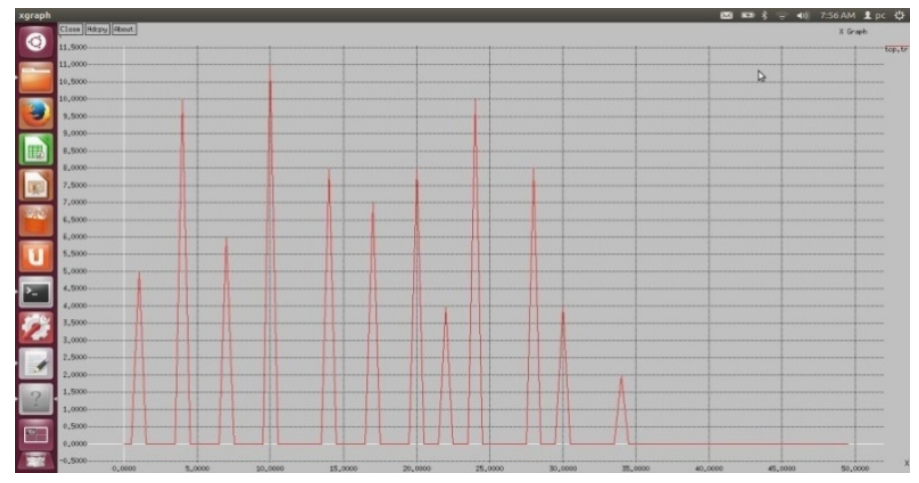

Figure 4.2: Graph for WSN using SCTP with packet loss

The communication is performed in the wireless sensor network using MPTCP with packet loss. Some packets don't reach at the destination due to loss. Loss may occur due to any reason. The performance graph for WSN using MPTCP with packet loss is shown in fig 4.4. The entire requirement is not full fill during Communication.

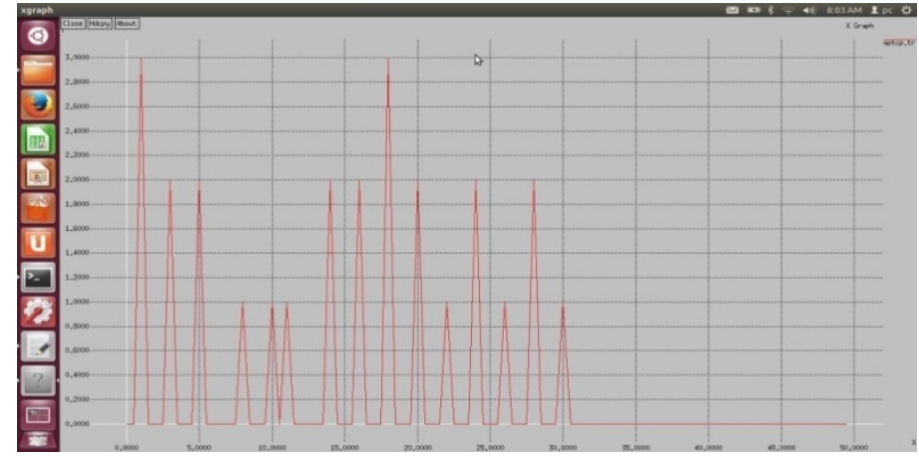

Figure 4.3: Graph for WSN using MPTCP with packet loss

\subsubsection{Comparison between TCP, SCTP and MPTCP with Packet Loss}

The performance analysis of wireless sensor network using all three approaches with packet loss is shown in fig. 4.5. The entire requirement is not full fill during communication in packet loss. MPTCP gives better performance than other approaches.

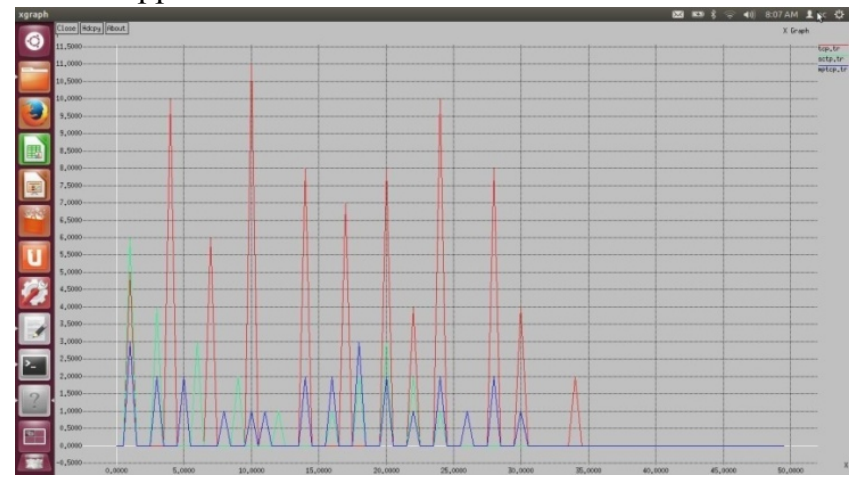

Figure 4.4:Comparison performance graph between TCP, SCTP and MPTCP with packet loss 


\section{CONCLUSION AND FUTURE WORK}

\subsection{Conclusion}

Transmission Control Protocol, Stream Control Transmission Protocol and Multipath TCP are used at the transport layer in wireless sensor network. It this work, the comparative study of SCTP, TCP and MPTCP is performed. To perform the comparison of protocols, Network Simulator-2 (NS2) tool used. A wireless sensor network designed. TCP, SCTP and MPTCP are compared for the packet loss and without packet loss environment. The MPTCP gives the better performance than the TCP and SCTP.

\subsection{Future Work}

In this work three protocols SCTP, TCP and MPTCP are compared under the assumption like fixed geographical area, constant energy dispersion, and fixed node position. In future the comparison can be done on the basis of the moving node in different geographical area. In this work defined energy

specification is considered. In future new algorithm can be developed for the dynamic position of nodes and variable energy dispersion. All these attempts contribute their important role in the improvement of wireless sensor network.

\section{References}

[1] Bhisham Sharma and Trilok C. Aseri. (2012). :A Comparative Analysis of Reliable and Congestion-Aware Transport Layer Protocols for Wireless Sensor Networks.International Scholarly Research Network Sensor Networks.

[2] P. Prittopaul, Dr.N. Shankarram. (2014, March). : Trust Aware Secure Routing for Cluster-based Wireless Sensor Networks - A Comparative Study. International Journal of Innovative Research in Computer and Communication Engineering. Proceedings of International Conference on Global Innovations in Computing Technology. ICGICT.

[3] Jennifer Yick, Biswanath Mukherjee, Dipak Ghosal. (2008). : Wireless sensor network survey (pp. 2292-2330).

[4] Ahmad Abed Alhameed Alkhatib, Gurvinder Singh Baicher. (2012). : Wireless Sensor Network Architecture. International Conference on Computer Networks and Communication System.IACSIT.

[5] Chonggang Wang, Kazem Sohraby, Bo Li, and Weiwen Tang. (2005, May). :Issues of Transport Control Protocols for Wireless Sensor Networks Communications Circuit Systems.Proceedings.(pp. $422-426$ ).

[6] Alakesh Braman, Umapathi G. R. (2014, February). : A Comparative Study on Advances in LEACH Routing Protocol for Wireless Sensor Networks: A survey. International Journal of Advanced Research in Computer and Communication Engineering. 
[7] K. Sundaresan, V. Anantharaman, H. Y. Hseeh, and R. Sivakumar. (2003, June). : ATP: a reliable transport protocol for ad-hoc networks. In Proceedings of the ACM Symposium on Mobile Ad Hoc Networking and Computing.(pp. 64-75). ACM.

[8] A. Dunkels, J. Alonso, and T. Voight. (2004). : Making TCP/IP Viable for wireless sensor networks.In Proceedings of European Workshop on Wireless Sensor Networks (pp. 1-4).

[9] Y. Zhou and M. R. Lyu. (2005). : PORT: a price-oriented reliable transport protocol for wireless sensor network. In Proceedings of 16th IEEE International Symposium on Software Reliability Engineering.(pp. 117-126).

[10] T. Braun, T. Voigt, and A. Dunkels. (2007, January). : TCP support for sensor networks.In Proceedings of the 4th Annual Conference on Wireless on Demand Network Systems and Services. ( pp. 162-169).

[11] B. Chellaprabha, Dr. S. Chenthur Pandian, Dr. C.Vivekanandan. (2012, Apr). :Performance of TCP, UDP and SCTP on Sensor Network with Different Data Reporting Intervals.IOSR Journal of Engineering.( pp. 621-628).

[12] Yung-Chih Chen, Don Towsley. (2014). : On Buffer bloat and Delay Analysis of Multi Path TCP in Wireless Networks.IFIP.

[13]N. Tezcan and W. Wang. (2007). : ART: an asymmetric and reliable transport mechanism for wireless sensor networks. International Journal of Sensor Networks.(pp. 34).

[14] S. Kim, R. Fonseca, P. Dutta et al. (2007, November). : Flush: a reliable bulk transport protocol for multihop wireless networks.In Proceedings of the 5th International Conference on Embedded Networked Sensor Systems. (pp. 351- 365). ACM.

[15] E. Giancoli, F. Jabour, and A. Pedroza. (2008, December). : CTCP: reliable transport control protocol for Sensor networks. In Proceedings of the International Conference on Intelligent Sensors, Sensor Networks and Information Processing. (pp. 493-498).

[16] H. Zhou, X. Guan, and C.Wu. (2008, May). : Reliable transport with memory consideration in wireless sensor networks. In Proceedings of the IEEE International Conference on Communications.(pp. 2819-2824).

[17] Gitanjali Shinde, Swati Joshi. (2012). : Wireless Sensor Network with DCDD. In International Conference on Information and Network Technology IPCSIT.(pp. 122126). 